\title{
Efeito agudo de exercício aeróbio intervalado de moderada intensidade nas funções cognitivas em idosas depressivas
}

Acute effect of moderate-intensity interval aerobic exercise on cognitive functions in depressed elderly women

Efecto agudo del ejercicio aeróbico de intervalo de intensidad moderada sobre las funciones cognitivas en mujeres de edad avanzada deprimidas

\author{
Kamilla Feitosa Pereira de Souza' \\ Carla Menêses Hardman' \\ Rebeka Vila Nova Gomes ${ }^{1}$ \\ Otávio Rodrigues' \\ Daniela Karina da Silva Ferreira' \\ André dos Santos Costa \\ Marisa Moreira Braga'
}

\section{Resumo}

O objetivo do estudo foi verificar o efeito agudo de exercício aeróbio intervalado de moderada intensidade nas funções cognitivas em idosas depressivas. Trata-se de um estudo pré-experimental conduzido com nove idosas depressivas. $\mathrm{O}$ treino foi constituído por 20 min. de atividades de coordenação motora, 30 min. de treinamento aeróbio intervalado e 10 min. de alongamentos. As funções cognitivas avaliadas foram: controle inibitório (Stroop $T e s t)$; linguagem (fluência verbal) e capacidade de realizar ao mesmo tempo estímulos motor e cognitivo (dupla tarefa). Foram empregados teste te tamanho de efeito. Verificouse uma redução estatisticamente significativa no tempo médio despendido nos testes de Stroop $1(18,93 \pm 4,14 \times 14,82 \pm 2,17 ; 0,016 ; \mathrm{TE}=1,30)$, Stroop 2 (22,38 $\pm 5,23$ x 17,94 $\pm 3,03$; $0,016 ; \mathrm{TE}=1,08)$, Stroop $3(33,05 \pm 6,82 \times 27,05 \pm 5,38 ; 0,011 ; \mathrm{TE}=0,98)$ e tarefa cognitiva $(15,05 \pm 4,17 \times 11,71 \pm 3,71 ; 0,029 ; \mathrm{TE}=0,85)$. O exercício físico teve um efeito agudo no controle inibitório e no desempenho na dupla tarefa cognitiva em idosas depressivas.

Palavras - chave: Exercício. Cognição. Idoso.

\begin{abstract}
The objective of the study was to verify the acute effect of moderate intensity interval aerobic exercise on cognitive functions in depressed elderly women. This is a preexperimental study conducted with nine depressed elderly women. The training consisted of $20 \mathrm{~min}$. of motor coordination activities, $30 \mathrm{~min}$. of aerobic training interval and $10 \mathrm{~min}$. of lengthening. The cognitive functions evaluated were: inhibitory control (Stroop Test); language (verbal fluency) and ability to perform at the same time stimuli motor and cognitive (dual task). T-test and effect size were used. There was a statistically significant reduction in the average time spent on Stroop 1 tests $(18.93 \pm 4.14 \times 14.82 \pm 2.17$; 0.016; $\mathrm{TE}=1.30)$, Stroop $2(22.38 \pm 5.23 \times 17.94 \pm 3.03 ; 0.016 ; \mathrm{TE}=1.08)$, Stroop 3 (33.05 \pm $6.82 \times 27.05 \pm 5.38 ; 0.011 ; \mathrm{TE}=0.98)$ and cognitive task $(15.05 \pm 4.17 \times 11.71 \pm 3.71$; $0.029 ; \mathrm{TE}=0.85)$. Physical exercise had an acute effect on inhibitory control and performance on dual cognitive task in depressed elderly women.
\end{abstract}

Keywords: Exercise. Cognition. Elderly.

1. Departamento de Educação Física/Universidade Federal de Pernambuco - Grupo de Pesquisa em Atividade Física, Saúde e Qualidade de Vida. Autor de correspondência: Carla Menêses Hardman, carlamhardman@gmail.com . Este conteúdo utiliza a Licença Creative Commons Attribution 4.0 International License Open Access. This content is licensed under a Creative Commons attribution-type BY ISSN 2595 - 0096. 


\section{Resumen}

El objetivo del estudio fue verificar el efecto agudo del ejercicio aeróbico de intervalo de intensidad moderada sobre las funciones cognitivas en mujeres mayores deprimidas. Este es un estudio pre-experimental realizado con nueve mujeres mayores deprimidas. El entrenamiento consistió en $20 \mathrm{~min}$. de actividades de coordinación motora, $30 \mathrm{~min}$. de intervalo de entrenamiento aeróbico y $10 \mathrm{~min}$. de estiramientos. Las funciones cognitivas evaluadas fueron: control inhibitorio (prueba de Stroop); lenguaje (fluidez verbal) y capacidad para realizar estímulos motores y cognitivos (tarea dual) al mismo tiempo. Se utilizaron la prueba $\mathrm{T}$ y el tamaño del efecto (TE). Hubo una reducción estadísticamente significativa en el tiempo promedio dedicado a Stroop $1(18.93 \pm 4.14 \times 14.82 \pm 2.17 ; 0.016$; $\mathrm{TE}=1.30)$, Stroop $2(22.38 \pm 5.23 \times 17.94 \pm 3.03 ; 0.016 ; \mathrm{TE}=1.08)$, Stroop $3(33.05 \pm$ $6.82 \times 27.05 \pm 5.38 ; 0.011 ; \mathrm{TE}=0.98)$ y tarea cognitiva $(15.05 \pm 4.17 \times 11.71 \pm 3.71 ; 0.029$; $\mathrm{TE}=0.85)$. El ejercicio físico tuvo un efecto agudo sobre el control inhibitorio y el rendimiento en la tarea cognitiva dual en mujeres mayores deprimidas.

Palabras - clave: Ejercicio. Cognición. Ancianos.

\section{INTRODUÇÃO}

A depressão é um dos principais problemas de saúde do mundo ${ }^{1}$. Trata-se de um transtorno de humor caracterizado pela sensação prolongada de tristeza, autodesvalorização, sentimento de culpa ${ }^{2}$, capacidades diminuídas de sentir prazer e manter a concentração ${ }^{3}$, que refletem diretamente na qualidade de vida do indivíduo depressivo ${ }^{4}$.

Esta doença pode acometer indivíduos de qualquer faixa etária, classe social ou cultura ${ }^{2}$ e pode causar alterações psicológicas simples, como transtorno de humor, e perturbações psiquiátricas graves, até ao suicídio ${ }^{5}$. Há comprometimentos também nas funções cognitivas por meio de distúrbios na atenção, da função executiva, da aprendizagem e memória de indivíduos depressivos ${ }^{4}$.

A função cognitiva é considerada preditor da qualidade de vida, pois as perdas podem resultar em prejuízo no funcionamento físico, social e emocional $^{6}$, particularmente em pessoas idosas, devido ao processo natural de envelhecimento. Um declínio modesto acontece mesmo sem a presença de doenças? ${ }^{7}$ Essa decadência dá-se pela perda de tecidos nas regiões corticais e substância branca cerebral, causando uma perda neurônios ${ }^{8}$.

A manutenção das funções cognitivas é um pré-requisito para a independência e saúde dos idosos ${ }^{9}$. Leite et al. $^{6}$ afirmam que a manutenção das funções cognitivas podem ser feitas por meio de atividades de lazer manuais e coletivas (artesanais, culturais, artísticas e da dança), estimulando o suporte social e, consequentemente, a melhora do equilíbrio biopsicossocial do idoso.

Há vários mecanismos que explicam como o exercício físico pode interferir nas funções cognitivas. 
Antunes $^{10}$ afirma que o exercício libera hormônios como catecolaminas, vasopressina e adrenocorticotrófico (ACTH) em proporção a quantidade de estresse, que poderiam, mediante mecanismos reflexos, melhorar a consolidação da memória. O exercício físico também pode prevenir as desordens degenerativas do sistema nervoso central por acarretar um aumento da circulação sanguínea cerebral, fornecendo a síntese neurotrófica, agentes responsáveis pela criação de novos neurônios em várias áreas cerebrais ${ }^{11}$.

Estudos de revisão sistemática revelaram efeitos benéficos do exercício físico nas funções cognitivas em idosos $^{9,12,13}$. Entretanto, uma metánalise conduzida com adultos deprimidos não evidenciou um efeito benéfico significativo do exercício na função cognitiva ${ }^{4}$.

Em relação ao tipo de exercício físico, Guimarães et al. ${ }^{9}$ revelaram que tanto a pratica de exercícios aeróbios quanto resistidos apresentaram efeitos positivos no desempenho cognitivo de idosos. Ensaios clínicos indicaram que os exercícios intervalados de alta intensidade melhoraram o desempenho nas tarefas de funções executivas em 49 homens mais velhos saudáveis ${ }^{14}$ e nos testes de memória, atenção e função executiva em
10 pacientes (22 a 64 anos) de ambos os sexos com esquizofrenia ${ }^{15}$. No entanto, Lamb et al. $^{16}$ verificaram que $\mathrm{o}$ treinamento com exercícios aeróbicos e de força com moderada a alta intensidade não foram suficientes para provocar melhorias significativas no desempenho cognitivo de idosos.

É importante mencionar que as medidas e os testes empregados para avaliar as funções cognitivas nos experimentos supracitados divergem. Engh et al. ${ }^{15}$ usaram o Matrics Consensus Cognitive Battery (MCCB), desenvolvido para avaliação de sujeitos esquizofrenicos. Lamb et al. ${ }^{16}$ utilizaram a Escala de Avaliação da Doença de Alzheimer - Subescala cognitiva (ADASCog). Bell et al. ${ }^{14}$, por sua vez, usaram Montreal Cognitive Assessment (MoCA) e Teste de Aprendizagem AuditivoVerbal de Rey (RAVLT). Diante do exposto, o presente estudo tem objetivo analisar o efeito agudo do exercício físico aeróbico intervalado de moderada intensidade na função cognitiva em idosas depressivas.

\section{MATERIAIS E MÉTODOS}

Trata-se de um estudo préexperimental derivado do projeto de pesquisa intitulado "Efeito do treinamento aeróbio na função cognitiva e na sintomatologia da depressão em 
idosas". Este projeto foi aprovado pelo Comitê de Ética em Pesquisa do Centro de Ciências da Saúde da Universidade Federal de Pernambuco - UFPE (no 1.873.471) e todas as participantes assinaram o Termo de Consentimento Livre e Esclarecido (TCLE).

A população alvo do presente estudo foi constituída por idosas com o diagnóstico de depressão do Recife-PE, Brasil. Foram excluídas pacientes que apresentaram alguma deficiência física e/ou relataram histórico de doenças neurodegenerativas. As idosas que participaram do estudo foram previamente diagnosticadas por médicos habilitados tanto da rede pública quanto privada de saúde do Recife, com base no Manual de Diagnóstico e Estatística de Distúrbios Mentais (DSM-V) e no Código Nacional de Doenças (CID-10). As idosas possuíam idade igual ou superior a 60 anos e não tinham praticado exercício físico por pelo menos seis meses. Para o recrutamento das participantes foram utilizados veículos de comunicação e encaminhamento de profissionais habilitados. As participantes foram selecionadas a partir de demanda espontânea.

O protocolo de treino foi constituído por 20 minutos de aquecimento com atividades de coordenação motora para membros superiores e inferiores, estimulando a bilateralidade e o próprio movimento realizado na caminhada (exemplo, caminhar com uma mão apontando para cima e a outra para o lado, trocando os movimentos das mãos conforme troca a passada); 30 minutos de exercício aeróbio intervalado de moderada intensidade (6 estímulos de 1 min a $75 \%$ do $\mathrm{VO}_{\text {2Max }}$ por 4 min a $\sim 55 \%$ do $\left.\mathrm{VO}_{2 \max }\right) ; 10$ minutos de volta a calma com alongamentos (sentadas) e exercícios de relaxamento (mantendo a atenção na respiração e com a cadência de dois segundos de inspiração e expiração). Toda a sessão de exercício foi acompanhada por músicas escolhidas pelas idosas.

$\mathrm{O}$ treinamento foi realizado $\mathrm{em}$ uma quadra coberta e a intensidade foi controlada pela percepção subjetiva de esforço (escala de BORG - CR10). As ancoragens das intensidades individuais percebidas para o estímulo e recuperação na sessão intervalada e a familiarização com a esteira ergométrica foram estabelecidas em sessão prévia. As idosas foram submetidas a um treinamento objetivado em esteira ergométrica com demandas metabólicas para o estímulo e a recuperação estimados pela equação do Colégio Americano de Medicina do Esporte $^{17}$ para caminhada $\left(\mathrm{VO}_{2}=\mathrm{V} \times 0,1\right.$ $+\mathrm{V} \times 1,8 \times \%+3,5)$, utilizando o $\mathrm{VO}_{2 \operatorname{mix}}$ previamente determinado. As idosas 
Tabela 1. Dados descritivos (média e desvio padrão ou mediana e intervalo interquartil) e tamanho do efeito dos escores das funções cognitivas nas condições pré e pós-exercício em idosas com depressão.

\begin{tabular}{|c|c|c|c|c|c|}
\hline Variáveis & Pré & Pós & TE & Classificação & $\begin{array}{c}\text { Valor } \\
p^{*}\end{array}$ \\
\hline \multicolumn{6}{|l|}{ Controle Inibitório } \\
\hline Stroop 1 - Tempo médio de resposta (segundos) & $\begin{array}{l}18,93 \\
(4,14)\end{array}$ & $\begin{array}{l}14,82 \\
(2,17)\end{array}$ & 1,30 & $\begin{array}{l}\text { Muito } \\
\text { grande }\end{array}$ & 0,016 \\
\hline Stroop 1 - Mediana de erros & $0(0-1)$ & $0(0-0)$ & - & - & 0,157 \\
\hline Stroop 2 - Tempo médio de resposta (segundos) & $\begin{array}{l}22,38 \\
(5,23)\end{array}$ & $\begin{array}{l}17,94 \\
(3,03)\end{array}$ & 1,08 & Grande & 0,016 \\
\hline Stroop 2 - Mediana de erros & () $(0-2)$ & $0(0-2)$ & - & - & 0,317 \\
\hline Stroop 3 - Tempo médio de resposta (segundos) & $\begin{array}{l}33,05 \\
(6,82)\end{array}$ & $\begin{array}{l}27,05 \\
(5,38)\end{array}$ & 0,98 & Grande & 0,011 \\
\hline $\begin{array}{l}\text { Stroop } 3 \text { - Mediana de erros } \\
\text { Linguagem }\end{array}$ & $2(0-5)$ & $1(0-3)$ & - & - & 0,228 \\
\hline Fluência verbal - quantidade média de frutas & $\begin{array}{l}14,78 \\
(3,38)\end{array}$ & $\begin{array}{l}15,33 \\
(2,91)\end{array}$ & \multicolumn{2}{|c|}{-0,017Insignificante } & 0,430 \\
\hline \multicolumn{6}{|l|}{$\begin{array}{l}\text { Capacidade de realização motora e cognitiva } \\
\text { simultânea }\end{array}$} \\
\hline DTC - Tempo médio gasto (segundos) & $\begin{array}{l}15,05 \\
(4,17)\end{array}$ & $\begin{array}{l}11,71 \\
(3,71)\end{array}$ & 0,85 & Grande & 0,029 \\
\hline DTC - Mediana de erros & $1(0-3)$ & $0(0-2)$ & - & - & 0,047 \\
\hline DTC - Mediana de acertos & $2(0-5)$ & $3(0-5)$ & - & - & 0,038 \\
\hline DTM - Tempo médio gasto (segundos) & $\begin{array}{l}10,49 \\
(1,67)\end{array}$ & $9,51(1,23)$ & 0,68 & Médio & 0,115 \\
\hline DTV - Tempo médio gasto (segundos) & $9,93(1,05)$ & $\begin{array}{l}10,38 \\
(1,50)\end{array}$ & $-0,35$ & Insignificante & 0,405 \\
\hline
\end{tabular}

${ }^{*}$ Teste t para amostras dependentes. Legenda: $\mathrm{TE}=$ Tamanho do Efeito; EGD $=$ Escala Geriátrica de Depressão; DTC $=$ Dupla Tarefa Cognitiva; DTM $=$ Dupla Tarefa Motora; DTV $=$ Dupla tarefa visuoespacial.

foram solicitadas a reproduzir a intensidade desta atividade na esteira na sessão de treinamento em uma quadra do Núcleo de Educação Física e Desportos da UFPE. A intervenção foi realizada em grupo e orientada por estudantes e profissionais de Educação Física.

Após assinatura do TCLE, as idosas realizaram uma anamnese para obter informações sociodemográficas e aspectos de saúde como: idade, escolaridade, estado civil, medicamentos e histórico de doenças. No período pré e pós-intervenção foram aplicados a Escala de Depressão Geriátrica (EDG-30) para acompanhar os sintomas depressivos. Esta escala possui 30 questões fechadas, com resposta afirmativa ou negativa, com um ponto para cada resposta compatível com os sintomas depressivos ${ }^{18}$.

As funções cognitivas avaliadas foram: atenção e controle inibitório (Stroop Test) ${ }^{19}$; linguagem (fluência verbal $)^{20}$; e capacidade de realizar ao mesmo tempo estímulos motor e cognitivo (Dupla Tarefa) ${ }^{21}$.

Anteriormente ao trabalho de campo, foi elaborado um manual de coleta de dados e conduzidos treinamentos com o objetivo de uniformizar os protocolos de aplicação dos testes cognitivos e a intervenção propriamente dita. As informações contidas nos instrumentos foram 
tabuladas no programa Microsoft ${ }^{\circledast}$ Office Excel (versão 2007).

Para análise descritiva das variáveis quantitativas foram utilizados média e desvio padrão. A normalidade da distribuição dos dados foi verificada pelo teste de Shapiro-Wilk. Para verificar o efeito agudo do exercício aeróbio intervalado nas funções cognitivas foi empregado teste $t$ para amostras dependentes. Em complemento, foram realizadas análises do tamanho de efeito ${ }^{22}$ da média e dos desvios padrão de ambos os grupos (estimativa $\mathrm{d}_{\mathrm{m}}$ de Cohen), classificando os valores em: insuficiente $(<0,19)$; pequeno $(0,20$ a 0,49$)$; médio (0,50 a 0,79); grande (0,8 a 1,29); muito grande $(>1,30)$. Para efetuar as análises dos dados foi utilizado o programa SPSS (versão 16) e foi adotado um valor de significância de $\mathrm{p}<0,05$.

\section{RESULTADOS E DISCUSSÃO}

Das 14 idosas depressivas selecionadas, uma não concluiu as avaliações iniciais e quatro não completaram o período de intervenção e consequentemente não realizaram as avaliações finais. Portanto, participaram do estudo nove idosas com diagnóstico de depressão, com idade entre 60 e 75 anos (66,3 $\pm 4,5$ anos). Identificou-se que oito idosas eram aposentadas e quatro tinham mais de 10 anos de escolaridade.
Em relação ao estado civil, cinco eram separadas, duas solteiras, uma viúva e uma casada, e seis referiram ter filhos.

Ao ser verificado o tempo de diagnóstico da depressão, identificou-se que quase todas as idosas tinham um ano ou mais de acompanhamento clínico, exceto uma que tinha um mês. Apenas duas alegaram usar algum medicamento para o tratamento da depressão. Além disso, não foi verificada uma redução nos escores médios de depressão (EDG-30) quando comparada as condições pré e pós-intervenção $\quad(11,33 \pm 5,79 \quad$ versus $12,67 \pm 6,91 ; \mathrm{p}=0,111)$. Os resultados do efeito do exercício aeróbio nas funções cognitivas em idosas com depressão nas condições pré e pós-intervenção estão apresentados na Tabela 1 .

\section{CONCLUSÃO}

O presente estudo verificou efeito agudo positivo no controle inibitório e no desempenho da dupla tarefa cognitiva após realização de exercício aeróbio intervalado de moderada intensidade em idosas com depressão. Na pratica, isso parece indicar que as idosas apresentaram menor grau de distração após uma sessão de exercício físico.

\section{REFERÊNCIAS}

1. World Health Organization (WHO). Depression. [Internet]. Geneva: WHO, 2018; [citado em 2019 set 17]. Disponível em: https://www.who.int/news-room/factsheets/detail/depression. 
2. Oliveira DG, Fortes LS, Evangelista WLS, Scoss DM. Os benefícios da atividade física para indivíduos portadores de depressão. UniÍtalo em Pesquisa 2016:6(2): 90-110.

3. Fonseca AA, Coutinho MPL, Azevedo RLW. Representações sociais da depressão em jovens universitários com e sem sintomas para desenvolver a depressão. Psicol-Reflex Crit 2008;21(3):492-8.

4. Brondino N, Rocchetti M, Fusar-Poli L, Codrons E, Correale L, Vandoni M, et al. A systematic review of cognitive e? ects of exercise in depression. Acta Psychiatr Scand 2017;135(4):285-95.

5. Coutinho MPL, Gontiès B, Araújo LF, Sá RCN. Depressão, um sofrimento sem fronteira: representações sociais entre crianças e idosos. Psico-USF 2003;8(2):93-2.

6. Leite MT, Winck MT, Hildebrandt LM, Kirchner RM, Silva LAA. Qualidade de vida e nível cognitivo de pessoas idosas participantes de grupos de convivência. Rev. Bras. Geriatr. Gerontol. 2012;15(3):481-92.

7. Yassuda MS, Lasca VB, Neri AL. Metamemória e auto-eficácia: um estudo de validação de instrumentos de pesquisa sobre memória e envelhecimento. Psicol. Refl Crít 2005;18(1):78-90.

8. Colcombe, SJ et al. Aerobic fitness reduces brain tissue loss in aging humans. J Gerontol, 2003;58(2):176-80.

9. Guimarães AV, Rocha SV, Barbosa AR. Exercise and cognitive performance in older adults: a systematic review. Medicina 2014;47(4):377-86.

10. Antunes HKM. A influência do exercício físico aeróbico em funções cognitivas e viscosidade do sangue de idosos normais [Dissertação]. São Paulo:
Universidade Federal de São Paulo; 2003.

11. Banhato EFC, Scoralick NN, Guedes DV, Silva KCA, Mota MMPE. Atividade fisica, cognição e envelhecimento: estudo de uma comunidade urbana. Psicol. teor. prat. 2009;11(1):76-84.

12. Braga MM, Soares FC, Costa AS, Deslandes AC, Hardman CM. Efeito do exercício físico nas funções cognitivas em idosos depressivos: revisão sistemática. ConScientiae saúde. 2019;18(1):141-8.

13. Carvalho A, Rea IM, Parimon T, Cusack BJ. Physical activity and cognitive function in individuals over 60 years of age: a systematic review. Clin Interv Aging 2014;9:661-82.

14. Bell KE, Fang H, Snijders T, Allison DJ, Zulyniak MA, Chabowski A, et al. A multi-ingredient nutritional supplement in combination with resistance exercise and high-intensity interval training improves cognitive function and increases $n-3$ index in healthy older men: a randomized controlled trial. Front Aging Neurosci. 2019;11(107):1-13.

15. Engh JA, Andersen E, Holmen TL, Martinsen EW, Mordal J, Morken G, et al. Effects of high-intensity aerobic exercise on psychotic symptoms and neurocognition in outpatients with schizophrenia: study protocol for a randomized controlled trial. BMC, 2015; 16(557):1-12.

16. Lamb SE, Sheehan B, Atherton N, Nichols V, Collins H, Mistry D, et al. Dementia and physical activity (DAPA) trial of moderate to high intensity exercise training for people with dementia: randomised controlled trial. BMJ 2018;361(k1675):1-11.

17. Thompson PDR, Pescatello DLS, Arena R. Diretrizes do ACSM - para os testes de esforço e sua prescrição: Guanabara Koogan, 2014. 
18. Sousa R, Medeiros JGM, Moura ACL, Souza CLM, Moreira IF. Validade e fidedignidade da Escala de Depressão Geriátrica na identificação de idosos deprimidos em um hospital geral. J. bras. psiquiatr. 2007;56:102-107.

19. Strauss E, Sherman EMS, Spreend OA. Compendium of neuropsychological tests: Administration, norms, and commentary. 3rd ed. Oxford, UK: Oxford University Press; 2006.

20. Brucki SM, Rocha MS. Category fluency test: effects of age, gender and education on total scores, clustering and switching in Brazilian Portuguesespeaking subjects. Braz J Med Biol Res. 2004;37(12):1771-7.

21. Silva RJM, Dias SMS, Piazza L. Desempenho em atividades de simples e dupla tarefas de idosos institucionalizados que realizam e não realizam fisioterapia. Fisioter. Pesqui. 2017;24:149-56.

22. Espírito-Santo H, Daniel F. Calcular e apresentar tamanhos do efeito em trabalhos científicos (1): As limitações do $\mathrm{p}<0,05$ na análise de diferenças de médias de dois grupos. RPICS. 2015;1(1):3-16.

23. Vasques PE, Moraes H, Silveira H, Deslandes AC, Laks J. Exercício agudo melhora a cognição em idosos deprimidos: o efeito de dupla tarefa. Clinicas 2011;66(9):1553-57.

24. Moreira AGG, Malloy-Diniz LF, Fuentes D, Correa H, Lage GM. Atividade física e desempenho em tarefas de funções executivas em idosos saudáveis: dados preliminares. Rev Psiq Clin. 2010;37(3):109-12.

25. Merege Filho CAA, Alves CRR, Sepúlveda CA, Costa AS, Lancha Júnior AH, Gualano B. Influência do exercício físico na cognição: uma atualização sobre mecanismos fisiológicos. Rev Bras Med Esporte. 2014;20(3):237-41.
27. Lima FAS. Efeito do exercício físico em meio aquático na memória e controle inibitório em idosas [Trabalho de Conclusão de Curso]. Departamento de Educação Física, Universidade Federal do Rio Grande do Norte, 2018.

Informações do artigo / Information of this article:

Recebido: 10/11/2019

Aprovado: 14/11/2019

Publicado: 26/03/2020

Received: 10/11/2019

Approved: 14/11/2019

Published: 26/03/2020

Carla Menêses Hardman

ORCID: 0000-0002-4355-0112.

Apoio / Acknowledgment

Agradecemos às idosas que se disponibilizaram a participar dessa pesquisa, aos monitores e a CAPES pelo auxílio financeiro.

Como citar esse artigo / How to cite this article:

De Souza, K. F. P. et al. Efeito agudo de exercício aeróbio intervalado de moderada intensidade nas funções cognitivas em idosas depressivas. Arq. Bras. Ed. Fís., Tocantinópolis, v. 2, n. 2, Ago./Dez., p. 27 - 35, 2019. 